\title{
Renal cell carcinoma after neuroblastoma: A case study and review of the literature
}

\author{
Brendan Wallace, ${ }^{*}$ Michael Organ, MD, FRCSC;; Scott Bagnell, MD, FRCSC,; Ricardo Rendon, MD, FRCSC;, \\ Jennifer Merrimen, MD, FRCPC
}

*Memorial University, Health Sciences Centre, St. John's, NL; 'Department of Urology, Health Sciences Centre, St. John's, NL; §Department of Urology, Saint John Regional Hospital, Saint John, NB; *Department of Urology, Dalhousie University, Halifax, NS

Cite as: Can Urol Assoc J 2015;9(5-6):E316-8. http://dx.doi.org/10.5489/cuaj.2564 Published online May 13, 2015.

\section{Abstract}

We present a case of renal cell carcinoma (RCC) arising in a 26-year-old patient with a history of neuroblastoma. RCC after a previous diagnosis of neuroblastoma is very uncommon, and there have only been 23 reported cases. Using the results of our patient workup, we hoped to determine whether there was a genetic predisposition or iatrogenic cause for the RCC. There is no clear explanation why neuroblastoma survivors are prone to developing RCC. However, genetic predisposition and previous treatment likely play a role. Since there have been few cases described, and few investigations into the genetics of this subtype of RCC, it remains important for individual cases to be added to the literature of this recently described and rare entity.

\section{Introduction}

Renal cell carcinoma (RCC) after a previous diagnosis of neuroblastoma is very uncommon. We present a case of RCC arising in a 26-year-old patient with a history of neuroblastoma.

\section{Case report}

A 26-year-old female, who as a child had a neuroblastoma, presented with gross hematuria. The cystoscopy was normal, but a magnetic resonance imaging (MRI) of her abdomen showed two enhancing renal masses: one in the right upper pole $(3.3 \times 3.0 \mathrm{~cm})$ and the other in the left lower pole $(6.6 \times 4.5 \mathrm{~cm})($ Fig. 1). Both were suspicious for RCC and a biopsy of the left-sided lesion confirmed it. Bilateral partial nephrectomies were performed.

Grossly, the left-sided mass showed a heterogeneous appearance with tan, yellow, solid and cystic areas (Fig. 2). The left-sided mass showed mixed morphology on light microscopy with clear, vacuolated, reticular and eosinophilic cells noted (Fig. 3). Oncocytoid and rhabdoid cells were also seen.

An external expert opinion confirmed that this lesion fell within the spectrum of lesions known as "RCC associated with neuroblastoma." The right-sided partial nephrectomy showed a papillary RCC, with no obvious unusual histologic features.

The patient was diagnosed with a neuroblastoma when she was 18 months old. She had a large tumour that displaced both kidneys and a bone scan had positive findings in the tibia and facial bones. The patient received chemotherapy consisting of melphalan for 22 weeks as per the Children's Cancer Study Group protocol 323P. Subsequently, the patient had a laparotomy revealing residual disease in the retroperitoneal area around the pancreas. Biopsies of this area were positive for malignant cells, for which she received radiation at 2 Gy in 20 fractions.

In early adulthood, the patient developed multiple other solid masses. At 25, she was being investigated for abdominal pain. Imaging studies revealed two abdominal masses. The patient had a total abdominal hysterectomy with bilateral salpingo-oophorectemy, and core biopsies to sample a mesenteric mass. There were multiple leiomyomas within the uterus and mesenteric lesions were compatible with intra-abdominal fibromatosis (desmoid tumour).

During her stay in hospital the patient had a colonoscopy for rectal bleeding, revealing multiple colonic and rectal polyps. All significant polyps were removed and proved to be tubular adenomas and hyperplastic polyps.

In March 2010, an MRI revealed a well-circumscribed lesion in the left lobe of the liver measuring $5.2 \times 3.7 \mathrm{~cm}$, suggesting an adenoma or focal nodular hyperplasia, which has remained stable on follow-up.

Due to the unusual medical history and multiple neoplasms, the patient was referred for a consultation with a medical geneticist. She is being evaluated for a hereditary multiple neoplasia syndrome, but the investigations are ongoing. 


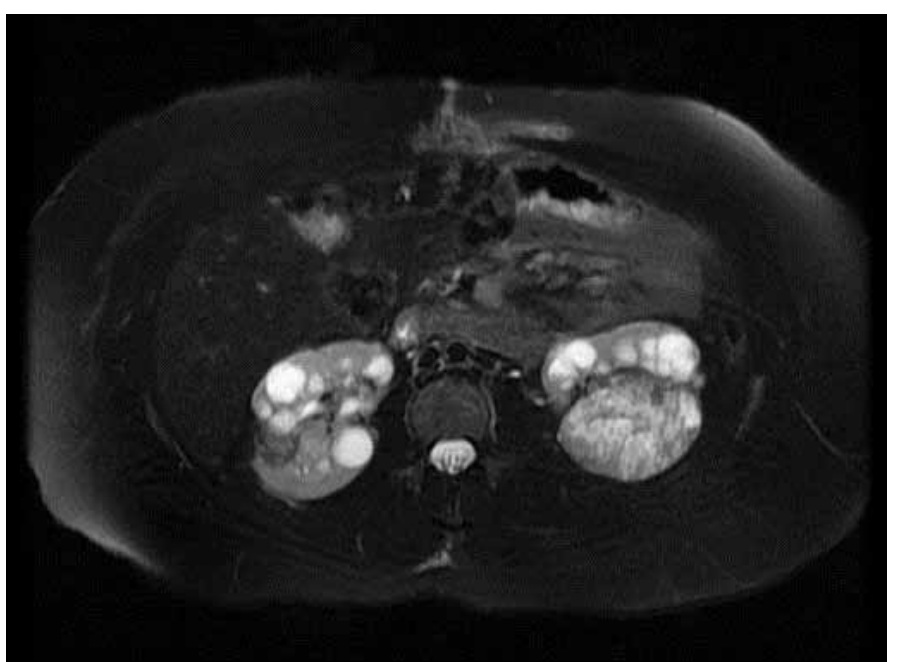

Fig. 1. A magnetic resonance image showing the left-sided renal mass. Multiple cysts are also present in the kidneys.

\section{Discussion}

RCC in neuroblastoma survivors is a rare RCC subtype..$^{1-3}$ There have only been 23 reported cases. ${ }^{4,5}$ Some patients developed RCC in the early years of follow-up after their neuroblastoma, while most develop RCC years later as young adults. ${ }^{6}$ This variation in the timeline for developing RCC suggests that the etiology and carcinogenesis of these are complex. RCC developing early after the detection and treatment of childhood neuroblastoma may be related to an inherited genetic mechanism, while RCC arising greater than 10 years after treatment may suggest that chemotherapy and radiation may have played a role.

The overall risk of a second malignancy is increased after chemotherapy and radiation for any cancer, ${ }^{7}$ but the risk of RCC is particularly increased after childhood malignancies. ${ }^{8}$ Most patients who go on to develop RCC have had chemotherapy, radiation, or both, as treatment for their neuroblastoma. However, only cisplatin and renal-directed radiotherapy of 5 Gy or greater have demonstrated an increased risk of developing RCC post-neuroblastoma. ${ }^{8}$

Our patient received a total dose of 40 Gy during her radiotherapy treatment, and her chemotherapy regimen consisted of mephalan. Radiation therapy may have played a role in the development of this patient's RCC. However, there are reports of similar lesions in patients who did not receive radiotherapy as part of their childhood cancer treatment. ${ }^{2}$

There is morphologic variation in the RCCs reported in survivors of neuroblastoma, including papillary morphology, clear cell morphology, anaplastic morphology, and oncocytoid or eosinophilic features. This pleomorphism in histologic appearance suggests that these lesions may arise via many different pathways. Studies looking at molecular pathways involved in RCC post-neuroblastoma have not elucidated a robust molecular explanation for these tumours; although,

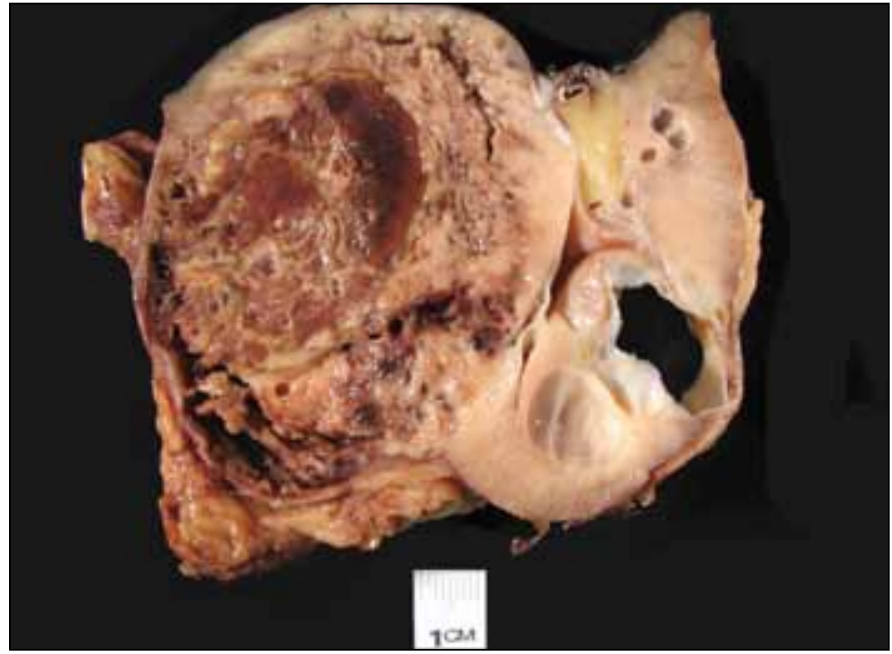

Fig. 2. Gross image of the left-sided partial nephrectomy specimen. Right aspect of image shows backgound kidney with acquired cysts. A 6.7-cm heterogenous tan, hemorrhagic, solid and cystic mass arising from the renal cortex is present.

an Xp11 translocation has been shown in some of these RCC cases. ${ }^{4}$ Curry and colleagues showed RCC with strong and diffuse reactivity of their RCC biopsy for Tp53, which is a known pathogenic mutation for cancer. ${ }^{9}$ Another genetic theory is the SDHB mutation, which is more often associated with paraganglioma. This relationship has been inferred by examining the genealogy of a particular family. ${ }^{10}$

In the previously described reports of RCC post-neuroblastoma, multiple other malignancies have been rare. Fleitz and colleagues reviewed 16 cases of RCC after neuroblastoma, with only one of these patients having another neoplasm, which was pheochromocytoma. Two more case studies by Schimke, Collins, and Stolle; ${ }^{10}$ and Flaig and colleagues ${ }^{5}$ showed multiple neoplasms in their patients.

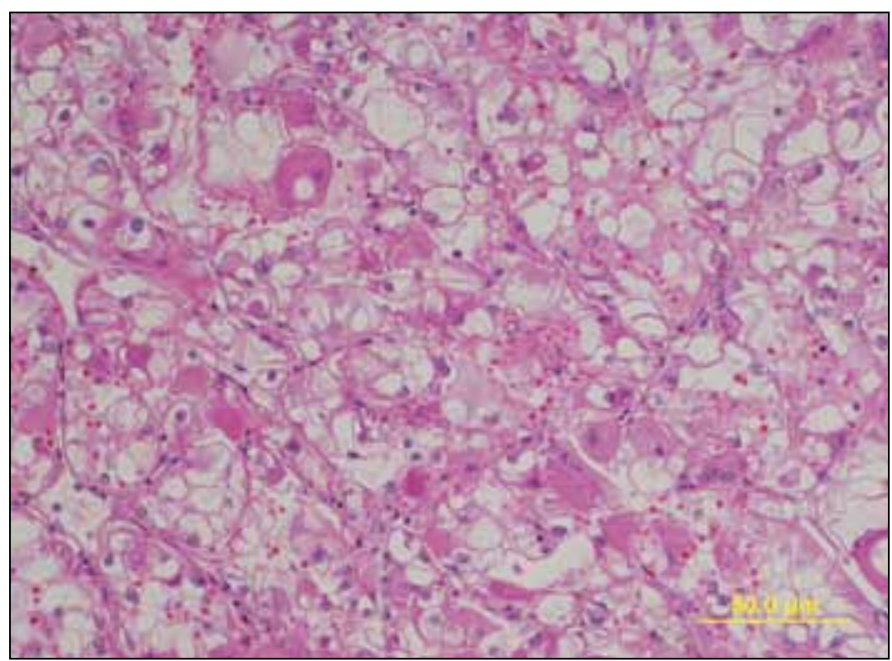

Fig. 3. A representative hematoxylin and eosin image of the morphology of the renal cell carcinoma in this patient $(\times 200)$. 
Wallace et al.

\section{Conclusion}

RCC after neuroblastoma is a recently described subtype of RCC. The reason neuroblastoma survivors are prone to developing RCC is unknown; however, genetic predisposition, previous chemotherapy and radiation treatment likely play a role. Since there have been few cases described and few investigations into the genetics of this subtype of RCC, it remains important for individual cases to be described to add to the literature. Our case is also unusual since our patient developed a number of other neoplastic lesions in early adulthood. Importantly, clinicians caring for patients with previous childhood malignancies should be aware of the increased risk of developing second malignancies, particularly RCC, in adulthood.

Competing interests: Mr. Wallace, Dr. Organ, Dr. Bagnell, and Dr. Merrimen all declare no competing financial or personal interests. Dr. Rendon is a member of the Advisory Board and the Speakers bureau for Amgen, Astellas, Ferring and Janssen.

This paper has been peer-reviewed.

\section{References}

1. Srigley JR, Delahunt B, Eble JN, et al. The International Society of Urological Pathology (ISUP) Vancouver classification of renal neoplasia. Am J Surg Pathol 2013;37:1469-89. http://dx.doi.org/10.1097/ PAS.0b013e318299f2dl

2. Srigley JR, Delahunt B. Uncommon and recently described renal carcinomas. Mod Pathol 2009;22:S2-23. http://dx.doi.org/10.1038/modpathol.2009.70

3. Fleitz JM, Wootton-Gorges SL, Wyatt-Ashmead J, et al. Renal cell carcinoma in long-term survivors of advanced stage neuroblastoma in early childhood. Pediatr Radiol 2003;33:540-5. http://dx.doi. org/10.1007/s00247-003-0913-x

4. Hedgepeth RC, Zhou M, Ross J. Rapid development of metastatic Xp 11 translocation renal cell carcinoma in a girl treated for neuroblastoma. J Pediatr Hematol Oncol 2009;31:602-4. http://dx.doi.org/10.1097/ MPH.0b013e3181acd981

5. Flaig TW, Kondo KL, La Rosa FG, et al. A young woman with multiple kidney lesions. J Oncol 2010;24:2826.

6. Koyle MA, Hatch DA, Furness PD 3rd, et al. Long-term urological complications in survivors younger than 15 months of advanced stage abdominal neuroblastoma. J Urol 2001;166:1455-8. http://dx.doi. org/10.1016/50022-5347(05)65808-9

7. Laverdiere $C$, Cheung NK, Kushner BH, et al. Long-term complications in survivors of advanced stage neuroblastoma. Pediatr Blood Cancer 2005;45:324-32. http://dx.doi.org/10.1002/pbc.20331

8. Wilson CL, Ness KK, Neglia JP, et al. Renal cell carcinoma after childhood cancer: A report from the childhood cancer survivor study. J Natl Cancer Inst 2013;105:504-8. http://dx.doi.org/10.1093/inci/dif014

9. Curry S, Ibrahim F, Grehan D, et al. Rhabdomyosarcoma-associated renal cell carcinoma: A link with constitutional TP53 mutation. Pediatr Develop Path 2011;14:248-51. http://dx.doi.org/10.2350/1007-0871-CR.1

10. Schimke RN, Collins DL, Stolle CA. Paraganglioma, neuroblastoma, and a SDHB Mutation: Resolution of a 30-year-old Mystery. Am J Med Genet 2010;152A:1531-5. http://dx.doi.org/10.1002/aimg.a.33384

Correspondence: Mr. Brendan Wallace, Memorial University, Health Sciences Centre, 300 Prince Phillip Drive, St. John's, NL AlB 3V6; brendan.wallace@mun.ca 